Editorial

\title{
Synthesis and Biomedical Applications of Functional Polymers
}

\author{
Jianxun Ding $\mathbb{D},{ }^{1}$ Di Li $\mathbb{D},{ }^{1}$ Jingxiao Chen $\mathbb{D},{ }^{2}$ Chao Zhao $\mathbb{D},^{3}$ \\ and Parisa Pour Shahid Saeed Abadi $\mathbb{D}^{4}$ \\ ${ }^{1}$ Key Laboratory of Polymer Ecomaterials, Changchun Institute of Applied Chemistry, Chinese Academy of Sciences, \\ 5625 Renmin Street, Changchun 130022, China \\ ${ }^{2}$ Department of Pharmaceutics, School of Pharmaceutical Sciences, Jiangnan University, 1800 Pohu Avenue, Wuxi 214122, China \\ ${ }^{3}$ Department of Chemical and Biological Engineering, The University of Alabama, 505 Hackberry Lane, Tuscaloosa, AL 35487, USA \\ ${ }^{4}$ Department of Mechanical Engineering-Engineering Mechanics, Michigan Technological University, 1400 Townsend Drive, \\ Houghton, MI 49931, USA
}

Correspondence should be addressed to Jianxun Ding; jxding@ciac.ac.cn

Received 28 July 2021; Accepted 28 July 2021; Published 13 August 2021

Copyright (c) 2021 Jianxun Ding et al. This is an open access article distributed under the Creative Commons Attribution License, which permits unrestricted use, distribution, and reproduction in any medium, provided the original work is properly cited.

In recent years, natural and synthetic biomedical polymers are receiving increasing attention due to their unique and variable chemical, physical, and biological properties. By improving the techniques of polymer synthesis and modification, functional polymers facilely achieve excellent biocompatibility and biodegradability and variable stimuliresponsiveness and bioactivity. Besides, functional polymers can be processed into various device modalities, such as nanoparticles, nanofibers, microspheres, microfibers, hydrogels, membranes, and scaffolds, to meet different practical applications.

Functional polymers have demonstrated promising applications in a wide range of biomedical fields, including disease diagnosis, therapeutic drug delivery, biosensors, tissue engineering, and medical devices. This special issue is aimed at systematically and comprehensively presenting the latest advances in developing preparation approaches and emerging biomedical applications of functional polymerbased materials, providing guidance and reference for future studies.

To meet this aim, the special issue collected sixteen research articles and two review articles. These articles cover the synthesis and biomedical applications of functional polymers.

Twelve research articles focused on polysaccharides and their applications in antitumor, antioxidation, and antiinflammatory functions. Antitumor activity is a potential property of polysaccharides, and nine different polysaccha- rides have been reported to exhibit antitumor activity against various tumors. M. Zeng et al. investigated the antitumor effect of Lycium barbarum polysaccharide (LBP) on human cutaneous squamous cell carcinoma and demonstrated that LBP induced the apoptosis of human cutaneous squamous cell carcinoma A431 cells by inhibiting autophagy and effectively inhibited tumor growth. Y. Zhang et al. confirmed that Codonopsis pilosula polysaccharide effectively inhibited the proliferation and motility of human hepatoma HepG2 cells in vitro possibly by inhibiting the $\beta$-catenin/TCF4 pathway. Similarly, M. Yao et al. illustrated that Laminaria japonica polysaccharide inhibited the survival of non-small-cell lung adenocarcinoma A549 cells through the same pathway. X. Du et al. found that Radix hedysari polysaccharide inhibited the growth of glioma U251 cells by cell cycle arrest and regulation of the tumor necrosis factor- $\alpha$ (TNF- $\alpha$ ) signaling pathway. X. Xu et al. demonstrated that sulfated polysaccharide from Undaria pinnatifida had antitumor activity against human prostate cancer DU145 cells, and the underlying mechanism may be related to inhibiting the migration and proliferation of cancer cells and inducing their apoptosis. W. Zhou et al. studied the effects of Polygonatum sibiricum polysaccharide (PSP) on the human esophageal squamous cell carcinoma Eca109 cells. The results showed that PSP significantly inhibited the proliferation, invasion, and migration of Eca109 cells and promoted cell apoptosis, and the underlying mechanism may be related to the inhibition of the nuclear factor $-\kappa \mathrm{B}(\mathrm{NF}-\kappa \mathrm{B})$ signaling pathway. 
Three research articles reported the synergistic antitumor efficacy of polysaccharides and other antitumor drugs. $X$. Liu et al. demonstrated that lentinan enhanced the cytotoxic effect of cisplatin on tumor cells, and the combined treatment of lentinan and cisplatin showed a satisfactory tumor inhibition effect in multiple tumor models. W. Wu et al. combined macrofungal polysaccharide (MFP) and vemurafenib to treat melanoma. The results showed that MFP significantly enhanced the tumor inhibitory effect of vemurafenib in human melanoma MeWo cell-bearing mice. $\mathrm{X}$. Yang et al. found that Ganoderma lucidum polysaccharide (GLP) improved the antitumor effect of 5-fluorouracil against gastric cancer BGC823 and SGC7901 cells by activating the natural killer group 2 member D (NKG2D)/MHC class I-related chain A (MICA) pathway.

Three research articles reported the applications of polysaccharides for treatment of other diseases, such as contrastinduced nephropathy, osteoarthritis, and intervertebral disc degeneration. M. Dai et al. demonstrated that the crude polysaccharide (cSFP-C) extracted from Sargassum fusiforme effectively prevented contrast-induced nephropathy due to their excellent antioxidant capacity. G. Lu et al. found that hyaluronic acid and Astragalus polysaccharide-capsuled nanoparticle effectively treated osteoarthritis by regulating cytokines and proteinase. Z. Zhai et al. confirmed that Polygonatum sibiricum polysaccharide (PSP) effectively reduced the apoptosis, inflammation, and oxidative stress of nucleus pulposus cells in rat with intervertebral disc degeneration, and it was a potential therapeutic drug for intervertebral disc degeneration.

Four original research articles report the synthesis and biomedical applications of other functional polymers. Q. Chen et al. prepared porous chitosan microsphere loaded with platelet-rich plasma and bone marrow-derived mesenchymal stem cells (PCM/PRP/BMSC composite), and they found that PCM/PRP/BMSC composite was beneficial for the repair of tibial defects. D. Patiño-Ruiz et al. synthesized chitosan-alginate nanodisk (Cs-Al ND) using the ionotropic gelation technique and demonstrated that Cs-Al ND was a promising drug delivery system with high encapsulation efficiency and controlled drug release. D. T. Nguyen et al. investigated the effect of microwave irradiation on poly(vinyl alcohol) (PVA) in $\mathrm{PVA} / \mathrm{Ag}^{+}$solution. The results confirmed that the redox reaction of $\mathrm{Ag}^{+}$with $\mathrm{PVA}$ and $\mathrm{Ag}^{+}$ reduced the hydrolysis rate of PVA. A. M. Díaz-Guerrero et al. used a mathematical model to evaluate the acetaminophen release in hydroxypropyl cellulose with polyacrylamide (HPC/PAAm) and confirmed that the proposed mathematical model was reliable.

In addition, two review articles summarized the latest advances in two different functional polymers for biomedical applications. G. Merkininkaite et al. introduced threedimensional laser lithography (3DLL) and the main categories of polymers used in 3DLL and then discussed the applications of 3DLL structural polymers in regenerative medicine. Finally, the current challenges and opportunities in this area were highlighted. Y. Yang et al. reviewed the preparation methods of the cellulose nanocrystal and its wide applications in various fields, which provided operable ideas and techniques for future high-end and ecofriendly functional composites.

In summary, this special issue bridges the synthesis methods, structure-property correlations, and biomedical applications of functional polymers and explores their underlying mechanisms. We hope to provide references for interested authors and inspire relevant researchers.

\section{Conflicts of Interest}

The authors declare no conflict of interest regarding the publication of this special issue.

\section{Acknowledgments}

The editors much appreciate the contributions of all the authors to the special issue and the constructive comments and valuable suggestions of all the reviewers.

Jianxun Ding

$\mathrm{Di} \mathrm{Li}$

Jingxiao Chen

Chao Zhao

Parisa Pour Shahid Saeed Abadi 